\title{
Use of performance indicators in the analysis of running gait impacts
}

\author{
Rosa Pàmies-Vilà, Francisco González, József Kövecses, Josep M. Font-Llagunes
}

This is a post-peer-review, pre-copyedit version of an article published in Multibody System Dynamics. The final authenticated version is available online at: https://doi .org/10.1007/s11044-017-9580-9. This document is licensed under a CC-BY-NC-ND license.

\begin{abstract}
Foot-ground impact is a critical event during the running cycle. In this work, three performance indicators were used to characterize foot-ground impact intensity: the effective preimpact kinetic energy, representative elements of the effective mass matrix, and the critical coefficient of friction. These performance indicators can be obtained from the inertial properties of the biomechanical system and its pre-impact mechanical state, avoiding the need to carry out force measurements. Ground reaction forces and kinematic data were collected from the running motion of an adult that adopted both rear-foot and fore-foot strike patterns. Different running cycles were analysed and statistical tests performed. Results showed that the three proposed indicators are able to illustrate significant differences between fore-foot and rear-foot strike impacts. They also support the hypothesis that fore-foot strike reduces impact intensity. On the other hand, a higher likelihood of slipping during the contact onset is associated with fore-foot strike pattern.
\end{abstract}

Keywords: Biomechanics, Running, Impact dynamics, Foot strike, Performance indicators

\section{Introduction}

Every year between $65 \%$ and $80 \%$ of all runners suffer injuries, which represents an important problem for the medical community [29]. Among the different phases of the running cycle, 
foot landing is probably the most critical event: the sudden loading of the lower extremities in contact with the weight-bearing surface produces a sharp rise of the normal ground reaction force. This impact is considered to be the main source of running-related injuries [38].

The foot strike pattern plays a key role in the development of foot-ground reaction forces. In practice, this pattern varies among runners; fore-foot and mid-foot strike gaits are more common when humans run barefoot or wearing minimal shoes, while shod runners usually tend to strike the ground with their heels. The experimental analysis presented in [24] showed that fore-foot strike (FFS) is associated with less intense collision forces than rear-foot strike (RFS). Accordingly, those who run barefoot tend to avoid heel-striking, and land instead on the toes or the middle part of the foot, thus decreasing the transient ground reaction force experienced at the contact onset.

Studying the differences between these foot strike patterns and considering the foot contact dynamics can be a key tool in the prevention of running injuries. Most dynamics studies on foot strike pattern are based on ground reaction forces (GRF). While FFS usually results in an attenuated impact peak in the GRF, RFS is characterized by a larger impact peak and a higher loading rate of the vertical GRF [6], [24]. Moreover, Lieberman et al. [24] showed that the loading rate for FFS barefoot running is about seven times lower than with barefoot RFS running.

Significant differences have been observed between FFS and RFS kinematics as well. De Wit et al. [9] used a four-segment human body model consisting of torso, thigh, shank, and foot to analyse the sagittal and frontal plane kinematics during the stance phase; the authors reported larger knee flexion angles and a more accentuated ankle plantar flexion just before contact in barefoot runners. Laughton et al. [23] found a considerably greater tibial acceleration in the FFS group and they also reported a lower peak knee flexion angle in FFS runners compared to RFS ones.

Some studies exist in the literature that intend to bridge the gap between foot-ground impact kinematics and dynamics. Biomechanics models of the human body are an important tool in this process. Lieberman et al. [24] and Kövecses and Kovács [22] used an L-shaped double pendulum that collided with the ground to represent the foot and leg in their kinematic and kinetic analyses. As an extension of [22], Zelei et al. [46] added the thigh and the trunk to the model and proposed another indicator for foot impact characterization, based on the velocity 
vector of the body centre of mass. A four-segment model of the lower extremity was used by Gerritsen et al. [14] to investigate the influence of muscle activation, position and velocities of body segments at touchdown and surface properties on impact forces during heel-toe running; Wright et al. [44] used a four-segment three-dimensional model to simulate impact in running with two different values of shoe hardness. These last two studies used forward-dynamics simulation techniques. The differences in muscle activity between FFS and RFS runners using a 3D lower extremity model were discussed more recently in [45].

Other authors have analysed the differences between RFS and FFS patterns from different points of view. Hanson et al. [17] used an oxygen analyser to compare the oxygen cost of barefoot running versus shod running. Squadrone et al. [42] used instrumented insoles to obtain the ground pressure distribution. Robbins et al. [37] employed a penetrometer to quantify the relation between localized load and pain and between load and depth of deformation. Divert et al. [10] used the EMG signals from five superficial lower leg muscles together with a treadmill dynamometer in order to further compare shod versus barefoot running. Low et al. [26], Maiwald et al. [28], and Belli et al. [5] used instrumented treadmills. However, biomechanical models were not used in any of these studies.

All the above-mentioned studies agree on the fact that the external loading rate is significantly larger in runners with RFS patterns, which translates into more intense foot-ground impacts. When it comes to quantifying impact intensity, however, only a few options are left to the researcher. Using direct GRF measurements is a possible one, which requires relying on the results provided by force plates or instrumented treadmills. It is also feasible to evaluate the impact force values from the system kinematics using any of the foot-ground contact force models that can be found in the literature, based on sphere, ellipsoid, cylinder, and plane contact geometries [25], [27], [31], [35]. With this method, however, all the parameters of the contact model, i.e., stiffness, damping and friction coefficients, and geometrical properties of the contact elements need to be identified and related to the actual contact phenomenon. An alternative approach is the use of kinematics-based, configuration-dependent performance indicators, obtained with biomechanical models [13]. These can provide relevant information about the way in which the running pattern influences the GRF, and how modifications in the gait of the runner can affect adversely or favourably the impacts during foot landing. With this method, force measurements or contact force models are not required. This was the approach followed in [13] [22] [46], and also in the present paper. 
The validity of a performance indicator is conditioned by the ability of the biomechanical model to capture the representative phenomena of the running motion; so far, only relatively simple representations of the lower limbs and the trunk have been used to this end, e.g., [24], [22], [46], [14]. In the present work, a detailed, full-body model of the runner was combined with motion-capture data and force-plate information to study impact dynamics with RFS and FFS gait patterns and to evaluate three selected performance indicators for impact intensity. Additionally, results obtained with this model were used to assess the suitability of partial human body representations to correctly evaluate the same indicators.

Three indicators are discussed in this work. The first one is the effective pre-impact kinetic energy, introduced in [15] as a way to estimate impact intensity in multibody systems that undergo collisions. This is a parametric performance indicator that can be used to investigate how changes in system configuration affect the peak force transmitted at an impact. The second indicator provides an alternative evaluation of impact intensity based on the components of the effective mass matrix along representative generalized directions [22]. Finally, the critical friction coefficient [12] was also selected as configuration-dependent indicator in this study. The value of this indicator represents the minimum coefficient of friction at the contact interface required to avoid the development of slip during impact, or to prevent sliding from reappearing once it has stopped.

The main contribution of this work is the use of these three indicators, obtained with a fullbody biomechanical model of the runner, to characterize the dynamics of foot-ground impact during running. The first two indicators account for the intensity of the generated forces during the impact interval, while the third one provides information about the safest system configurations with regard to avoiding slip at the contact interface. The indicators can be evaluated only with information about the system kinematics, i.e., generalized coordinates and velocities, and its inertial parameters. As a consequence, capturing the movement provides enough data to determine their values; a foot-ground contact force model is not necessary. The computational cost of evaluating the three proposed indicators is lower than that of other methodologies used in the studies cited before. 


\section{Materials and Methods}

\subsection{Experimental data}

The subject selected to perform the experiments was a 36-year old, healthy adult male, mass 72 $\mathrm{kg}$, and height $1.75 \mathrm{~m}$, free of injuries at the time of the experiments.
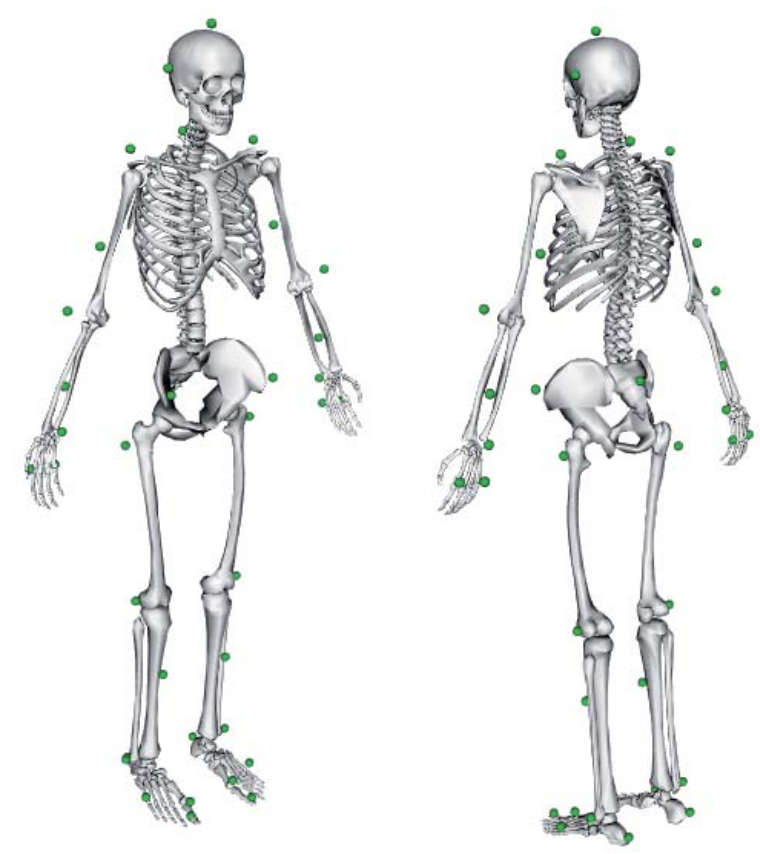

Figure 1: 3D view of the human skeleton with the set of 37 markers used.

In all the tests, the motion was captured by 18 Natural Point (Corvallis, USA) optical cameras sampling at $100 \mathrm{~Hz}$ that recorded the position of 37 reflective markers attached to the subject. The position of each marker on the human body followed, for the lower limbs, the marker set definition proposed by Vaughan et al. [43] and it was based on the set-up defined by Nigg [32] for the upper body (Fig. 1). Two series of experiments were performed:

- Series 1: Running motion on hard ground at the laboratory. In this set of tests, two force plates (AMTI AccuGait, Watertown, USA), sampled at $1000 \mathrm{~Hz}$, were embedded in the walkway and used to measure the foot-ground contact forces. The body motion was also captured by means of optical cameras.

- Series 2: Running on a non-instrumented NordicTrack (Logan, USA) treadmill at $8 \mathrm{~km} / \mathrm{h}$. In this series, the optical system captured the trajectories of the 37 markers, but no GRF measurements were recorded. The runner was given enough time to warm up and become 
familiar with the specific treadmill velocity.

In both series the tests were repeated until 15 acceptable trials for each strike pattern were obtained. The runner was barefoot in the FFS tests; he was shod during RFS tests.

It must be pointed out that several studies in the literature confirm that the kinetics of running on hard ground and on a treadmill are very similar. The overground and treadmill GRF curves have been qualitatively shown to be comparable, both for heelstrike and non-heelstrike runners [19]. Statistically significant differences may be observed in the time-histories of the joint moments and joint power [36]; however, the magnitude of these differences was found to be comparable to the variability in normal running parameters.

\subsection{Biomechanical model}

The human body was modelled as a multibody system made up of rigid links. The 2D model used consisted of 12 anatomical segments: trunk, head, two arms, two forearms, two thighs, two shanks and two hindfeet. The segments were connected by ideal revolute joints that defined a 14 degree-of-freedom (DOF) model.

Table 4 in Appendix A contains the anthropometric parameters of the model. The inertial properties of the segments were extracted, for the lower limbs, from a reduced set of measurements performed on the subject and by scaling published data according to his mass and height [43]. For the upper body, the inertial parameters of the model were obtained by scaling table data according to the mass and height of the subject [39], [41].

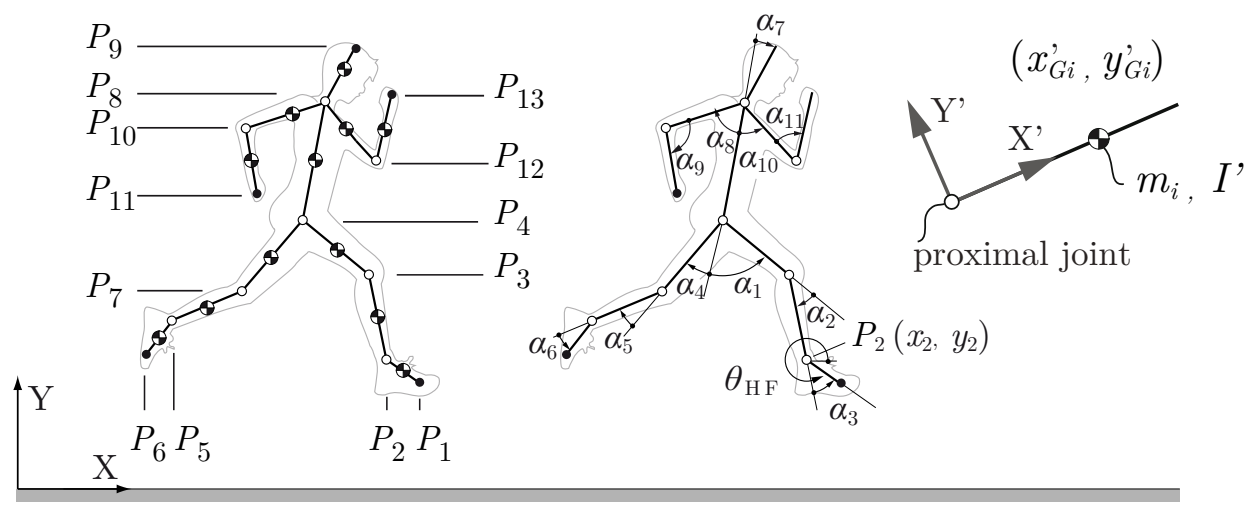

Figure 2: 2D biomechanical model of the subject. 
The full body model of the subject was developed with 38 dependent coordinates. These coordinates included a mix of Cartesian coordinates of points and angles representing either absolute or relative rotations of segments. The coordinate set was composed of the $X$ and $Y$ global coordinates of the 13 points that corresponded to the positions of the centres of all the revolute joints, along with the tips of five extreme segments representing the head, arms and feet, together with 12 angular variables. The first angle variable defined the orientation of the trunk with respect to the absolute reference frame, and the remaining ones specified the relative orientations of the body segments. The motion analysis was carried out using a set of $n=14$ independent coordinates $\mathbf{q}$ that comprised the two Cartesian coordinates of the centre of the left ankle joint $\left(x_{2}, y_{2}\right)$ in the treadmill reference frame and the 12 angles $\left(\theta_{H F}, \alpha_{1}, \ldots, \alpha_{11}\right)$ as shown in Fig. 2.

Regression equations [34] were used to determine the position history of the joint centres from the marker positions. However, the obtained data did not ensure the kinematic consistency and corrected values of the coordinates were determined using the optimization procedure described in [3]. Finally, an algorithm based on singular spectrum analysis (SSA) was applied to reduce the noise introduced by the kinematic consistency processing [2].

\subsection{Simplified biomechanical models}

Simpler biomechanical models can also be used to represent the body of the subject. Such partial body models can be found in several studies on running in the literature, e.g., [22], [9], [16], [45].

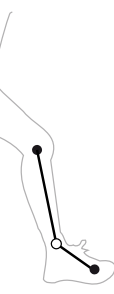

(a)

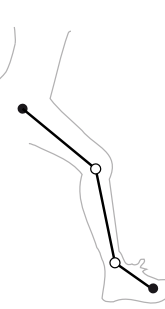

(b)

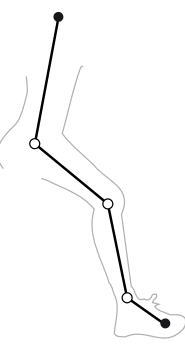

(c)

Figure 3: Partial models used: two-segment (a), three-segment (b), and four-segment (c) simplified models.

The characterization of the human body depends on the intended use of the model, and 
less detailed representations can suffice to capture representative aspects of the motion in some cases. Researchers can choose to use different numbers and types of body segments, joints, muscles, etc. depending on the purpose of their studies. In this work, three partial models were used to assess the effect of simplified modelling on the validity of the selected indicators. A two-segment model, composed of the foot that is in contact with the ground and its corresponding shank, was the simplest one. Three- and four-segment models were subsequently obtained adding to these two segments the thigh and the trunk, respectively, as shown in Fig. 3.

\subsection{Data Analysis}

Three performance indicators were defined and used to quantify impact intensity for each strike pattern, namely the effective pre-impact kinetic energy, the effective mass matrix, and the critical coefficient of friction, as it will be discussed in Section 3.

First, the ability of the indicators to quantify impact intensity had to be verified. These indicators were originally defined to characterize rigid-body, instantaneous impacts, during which the system configuration is assumed to remain without changes. In the case of foot-ground impact, the impact is not perfectly rigid as the bodies in contact are compliant; moreover, the system configuration does change between the beginning and the end of the stance phase. The GRF measurements obtained during the first series of experiments were used to confirm the validity of the indicators to determine impact intensity.
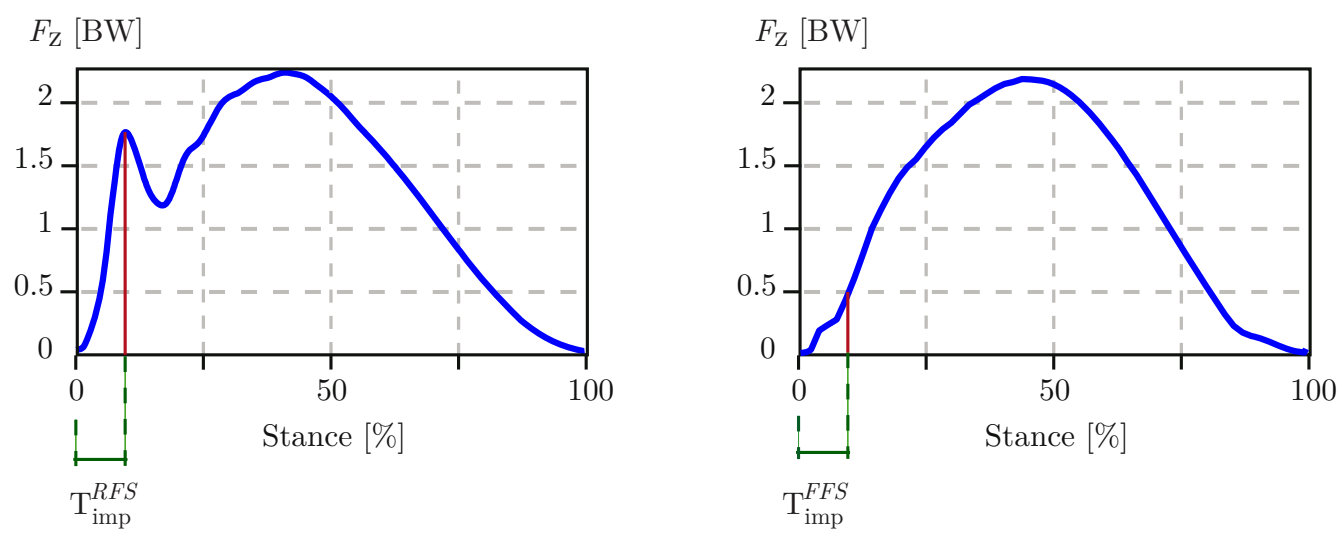

Figure 4: Vertical ground reaction force for the studied foot strike patterns, RFS (left) and FFS (right).

Fig. 4 shows the time-history of the normal ground reaction force $\left(F_{Y}\right)$, normalized and ex- 
pressed in body weights (BW), during two stance phases with RFS and FFS patterns respectively. These plots correspond to two of the 30 analysed samples, and are representative of the general behaviour observed in all of them.

In the RFS case, the normal reaction force features a clear impact transient spike; the maximum force value in this spike corresponds to the impact force, $F_{i m p}$, [24]. The rise time of $F_{i m p}$ defines the duration of the impact, $t_{i m p}^{R F S}$, which ranges between 18 and $35 \mathrm{~ms}$. The change in system configuration, i.e., the relative angles and displacements of the body segments with respect to each other, can be considered negligible between $t=0$ and $t=t_{i m p}^{R F S}$. In FFS the transient spike is not present, and $t_{i m p}^{F F S}$ was defined as the $8 \%$ of the stance phase, which is equivalent to the fraction of the stance that corresponds to $t_{i m p}^{R F S}$ in RFS running. Impact duration was found to be $t_{i m p}^{R F S}=0.03 \pm 0.002 \mathrm{~s}$ and $t_{i m p}^{F F S}=0.024 \pm 0.003 \mathrm{~s}$. The loading rate, $\gamma$, defined as the rate with which the impact force rose from $200 \mathrm{~N}$ to either $90 \%$ of its maximum (RFS) or until 6.3\% of the stance phase was reached (FFS), was also evaluated in both cases. This approach follows the method described in [24]. The values of $F_{i m p}$ and $\gamma$ were used to validate the proposed impact indicators.

In the processing of the experimental data, a gait event-detection algorithm [33] was implemented and applied to find the times at which foot-ground contact was established. This algorithm was based on the vertical velocity profile of the foot centre. In order to compare the two foot-strike patterns, a t-Student test was performed. This test is an inferential statistical tool that allows one to determine if there is a statistically significant difference between the means in two unrelated groups. The differences in the performance indicators between the 15 rear-foot impacts and the 15 fore-foot impacts were analysed.

The selected performance indicators were found to be highly dependent on the location of the foot-ground contact point. Therefore, parametric analyses were carried out to determine their variation with changes of the strike index, using the data provided by the second series of experiments. The strike index $S I$ is used to characterize different possible foot strike patterns. It describes the location of the centre of pressure $(\mathrm{CoP})$ at the beginning of the foot-ground contact with respect to the longitudinal axis of the foot. It is traditionally expressed as a percentage of the total foot length. Based on this index, runners can be classified as rear-foot, with a strike index between 0 and $33 \%$, mid-foot, $34 \%$ to $67 \%$, or fore-foot strikers, $68 \%$ to $100 \%$ [6].

In the literature, some studies represent the human body as a single particle (spring-mass 
models), or they assume that all the joints in the multibody model are locked at the time of impact [11], [30], [40], [22]. Other studies consider that all the velocities in the system are zero, except the vertical velocity component of a representative point [24], [22], [1], [8]. The way in which these hypotheses affect the proposed indicators was also analysed. Finally, the effect on the results of using the simplified body models described in Section 2.3 was studied as well.

\section{Indicators of impact intensity}

Using $n$ independent generalized coordinates $\mathbf{q}$, the motion of a mechanical system -the human body, in this case- can be described by the system of dynamics equations

$$
\mathbf{M}(\mathbf{q}) \ddot{\mathbf{q}}+\mathbf{c}(\mathbf{q}, \dot{\mathbf{q}})=\mathbf{f}
$$

where $\mathbf{M}$ is the $n \times n$ mass matrix, $\mathbf{c}$ represents the Coriolis and centrifugal effects, and $\mathbf{f}$ contains the generalized forces applied on the system.

Let us assume that each foot-ground impact is representable with a single-point contact model. The velocity of the contact point $Q, \mathbf{v}(Q)$, can be related to the generalized velocities $\dot{\mathbf{q}}$ through the $2 \times n$ Jacobian matrix $\mathbf{A}$ via the transformation

$$
\mathbf{v}(Q)=\left[\begin{array}{c}
v_{t}(Q) \\
v_{n}(Q)
\end{array}\right]=\mathbf{A} \dot{\mathbf{q}}=\left[\begin{array}{c}
\mathbf{A}_{t} \\
\mathbf{A}_{n}
\end{array}\right] \dot{\mathbf{q}}
$$

where $v_{t}$ and $v_{n}$ are the tangential and normal components of $\mathbf{v}(Q)$ with respect to the ground, which can be obtained from the generalized velocities $\dot{\mathbf{q}}$ through the $1 \times n$ Jacobian matrices $\mathbf{A}_{t}$ and $\mathbf{A}_{n}$, respectively.

The geometric parameters of the foot model are represented in Fig. 5; $P_{2}$ is the centre of the ankle joint and $P_{1}$ the distal point of the hindfoot segment. The distance between $P_{2}$ and $P_{1}$ is $l_{H F}$, while $l_{v}$ denotes the distance between $P_{2}$ and the foot sole, and $l_{h}$ is the total foot length. 


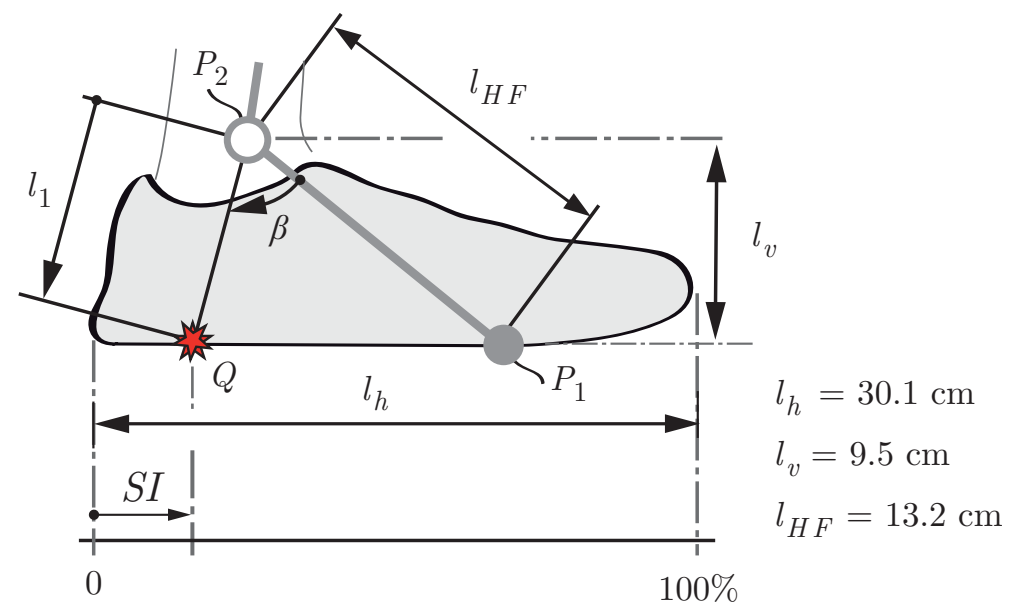

Figure 5: Foot and shoe model.

The expression of $\mathbf{A}$ takes the form

$$
\mathbf{A}=\left[\begin{array}{rrrrrr}
1 & 0 & -l_{1} \sin \left(\theta_{H F}-\beta\right) & 0 & \ldots & 0 \\
0 & 1 & l_{1} \cos \left(\theta_{H F}-\beta\right) & 0 & \ldots & 0
\end{array}\right]
$$

where $\theta_{H F}$ is the angle from the global $X$ axis to the line defined by points $P_{1}$ and $P_{2}$ as shown in Fig. $2, l_{1}$ is the distance between the contact point and the ankle, and $\beta$ is the angle from segment $P_{1}-P_{2}$ to line $Q-P_{2}$. Both $l_{1}$ and $\beta$ vary depending on the position of point $Q$. If the contact takes place at point $P_{1}$ on the fore-foot, $l_{1}=l_{H F}$ and $\beta=0$.

In this work, the duration of the foot-ground impact is considered to be negligible when compared to the time scale of the system dynamics; accordingly, the dynamics equations can be established following an impulse-momentum approach. The time instants that immediately precede and follow the contact onset are denoted by $t^{-}$and $t^{+}$, respectively; the corresponding sets of generalized velocities at these moments are $\dot{\mathbf{q}}^{-}$and $\dot{\mathbf{q}}^{+}$. The impact can then be represented by means of the introduction of a kinematic constraint at time $t^{+}$

$$
\mathbf{v}(Q)=\mathbf{A} \dot{\mathbf{q}}^{+}=\mathbf{0}
$$

which imposes the condition that the velocity of the impact point $Q$ becomes null when the contact is established. This constraint introduces reaction forces $\boldsymbol{\lambda}=\left[\begin{array}{cc}\lambda_{t} & \lambda_{n}\end{array}\right]^{\mathrm{T}}$, where $\lambda_{t}$ and $\lambda_{n}$ represent the tangential and normal ground reactions; the constrained dynamics can be 
expressed as

$$
\begin{aligned}
\mathbf{M} \ddot{\mathbf{q}}+\mathbf{c}=\mathbf{f}+\mathbf{f}_{c} & =\mathbf{f}+\mathbf{A}^{\mathrm{T}} \boldsymbol{\lambda} \\
\mathbf{A} \dot{\mathbf{q}} & =\mathbf{0}
\end{aligned}
$$

where $\mathbf{f}_{c}=\mathbf{A}^{\mathrm{T}} \boldsymbol{\lambda}$.

It can be assumed that internal joint actuation is finite, which means that ground contact reactions are the only impulsive forces in the system. Then, the impulse-momentum level dynamics equations can be written as

$$
\left|\frac{\partial T}{\partial \dot{\mathbf{q}}}\right|_{-}^{+}=\mathbf{M} \Delta \dot{\mathbf{q}}=\overline{\mathbf{f}_{c}}=\mathbf{A}^{\mathrm{T}} \bar{\lambda}
$$

where $T$ is the kinetic energy of the system, $\Delta \dot{\mathbf{q}}=\dot{\mathbf{q}}^{+}-\dot{\mathbf{q}}^{-}$is the velocity change during impact, and $\overline{\mathrm{f}_{c}}$ and $\bar{\lambda}$ represent the impulse of the generalized reaction forces. It is assumed that the duration of the impact is negligible with respect to the time-scale of the system dynamics. Accordingly, the system configuration is assumed to remain unchanged during the impact interval.

\subsection{Effective pre-impact kinetic energy}

The kinetic energy associated with the subspace of constrained motion defined by Eq. (2), $T_{c}$, can be used as an indicator to characterize impact intensity. It was reported in [21] that the preimpact value of $T_{c}$ is proportional to the impulse of the reactions. Experimental measurements in [15] showed that this quantity can be used as an indicator of the peak contact force developed during impact. The pre-impact kinetic energy associated with the subspace of constrained motion (SCM), or effective pre-impact kinetic energy, can be derived as

$$
T_{c}^{-}=\frac{1}{2}\left(\dot{\mathbf{q}}^{-}\right)^{\mathrm{T}} \mathbf{P}_{c}^{\mathrm{T}} \mathbf{M} \mathbf{P}_{c} \dot{\mathbf{q}}^{-}
$$

where $\mathbf{P}_{c}$ is the projector matrix onto the subspace of constrained motion [20] [13]

$$
\mathbf{P}_{c}=\mathbf{M}^{-1} \mathbf{A}^{\mathrm{T}}\left(\mathbf{A} \mathbf{M}^{-1} \mathbf{A}^{\mathrm{T}}\right)^{-1} \mathbf{A}
$$


In order to compare $T_{c}^{-}$in different kinematic conditions of impact, the dimensionless indicator $\xi$ was defined [21] as

$$
\xi=\frac{T_{c}^{-}}{T^{-}}=\frac{\left(\dot{\mathbf{q}}^{-}\right)^{\mathrm{T}} \mathbf{P}_{c}^{\mathrm{T}} \mathbf{M} \mathbf{P}_{c} \dot{\mathbf{q}}^{-}}{\left(\dot{\mathbf{q}}^{-}\right)^{\mathrm{T}} \mathbf{M} \dot{\mathbf{q}}^{-}}
$$

This indicator represents the fraction of pre-impact kinetic energy which is contained in the subspace of constrained motion associated with the impact.

\subsection{Effective mass matrix}

The effective mass is another indicator that can be used to characterize impact intensity. Several definitions of this parameter have been proposed in the literature; some of them require the knowledge of the contact force during the impact, e.g., see [7] and [1]. Others can be evaluated based on the system configuration, mass and inertia properties, and kinematic quantities. Among the latter, two expressions were selected in this research.

The first one was term $\mathbf{P}_{c}^{\mathrm{T}} \mathbf{M} \mathbf{P}_{c}$ in Eq. (8), which expresses the relation between the preimpact velocity and the effective pre-impact kinetic energy, $T_{c}^{-}$. This can be seen as the effective mass matrix of foot touchdown, $\mathbf{M}_{e f f}^{\mathbf{q}}$, associated with the parametrization of the system motion given by $\dot{\mathbf{q}}$ [22]. With the definition of $\mathbf{P}_{c}$ in Eq. (9), the effective mass matrix can be expressed as

$$
\mathbf{M}_{e f f}^{\mathbf{q}}=\mathbf{A}^{\mathrm{T}}\left(\mathbf{A} \mathbf{M}^{-1} \mathbf{A}^{\mathrm{T}}\right)^{-1} \mathbf{A}
$$

Another option is to obtain the effective mass matrix associated with the local parametrization of the impact, given by $\mathbf{v}(Q)$. The expression of this term is [15]

$$
\mathbf{M}_{\text {eff }}^{\mathbf{v}}=\left(\mathbf{A} \mathbf{M}^{-1} \mathbf{A}^{\mathrm{T}}\right)^{-1}
$$

The use of the effective mass matrix as indicator of impact intensity was reported in [22] using a foot-shank model. The system velocities in the study were set to zero, except for the ankle velocity component along the vertical direction. This made it possible to use as indicator a single scalar value, namely the element of the effective mass matrix that corresponded to this velocity component. Ref. [22] also reported that the existence of a horizontal component in the 
pre-impact foot velocity could change significantly the impact intensity, so the validity of the indicator in the general case still needs to be assessed.

According to [18], $\mathbf{M}_{\text {eff }}^{\mathbf{q}}(i, i)$, also termed locked effective inertia, represents the effective inertia that corresponds to generalized velocity $\dot{q}_{i}$ when all other coordinate directions are locked. Given the expression of the Jacobian matrix in Eq. (3), if the normal velocity at contact point $Q$ is the only non-zero velocity component in the system, i.e., the remaining DOFs are locked, the effective inertia related to the vertical velocities of the ankle and that of the contact point have the same expression. Therefore, $\mathbf{M}_{\text {eff }}^{\mathbf{v}}(2,2)=\mathbf{M}_{\text {eff }}^{\mathbf{q}}(2,2)=m_{\text {eff }}$. In the present paper, this element was selected as indicator of the impact intensity. A dimensionless indicator, $\chi$, was obtained dividing $m_{e f f}$ by the total mass of the subject, $m_{s}$

$$
\chi=\frac{m_{e f f}}{m_{s}}
$$

\subsection{Critical coefficient of friction}

The critical coefficient of friction, $\mu_{c}$, defined in [12] as

$$
\mu_{c}=\left|\frac{\mathbf{A}_{t} \mathbf{M}^{-1} \mathbf{A}_{n}^{\mathrm{T}}}{\mathbf{A}_{t} \mathbf{M}^{-1} \mathbf{A}_{t}^{\mathrm{T}}}\right|
$$

can also be used as an indicator to characterize the foot-ground contact dynamics during running.

The critical coefficient of friction is a configuration-dependent parametric indicator that illustrates the tendency to develop slip at the contact interface. Slip will be developed at the foot-ground interaction point if the coefficient of friction $\mu$ of the surfaces in contact is smaller than $\mu_{c}$. The coefficient of friction between the ground and feet or shoes usually ranges between $\mu=0.3$, for surfaces covered with loose granules, and $\mu=1.5$, for artificial turf [32]. Running shoes usually present $0.8<\mu<1.1$. The treadmill mat used to perform the experiments was made from rubberised vinyl, which has a high coefficient of friction, and so slip at the foot-mat interface after foot landing was not observed during the tests. 


\section{Results}

\subsection{Experiment series 1: running on hard ground}

Experimental results obtained during the first series of tests showed that significant differences existed between the GRF values obtained with RFS and FFS patterns.
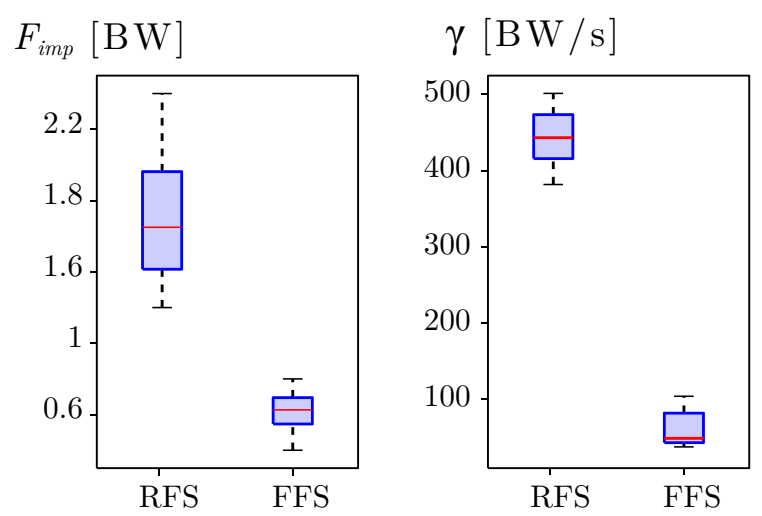

Figure 6: Force indices $F_{i m p}$ and $\gamma$ box plots obtained in experiment series 1.

The maximum impact force and loading rate in RFS experiments were $F_{i m p}=1.725 \pm 0.364$ BW and $\gamma=444.8 \pm 36.42 \mathrm{BW} / \mathrm{s}$. With FFS running they went down to $F_{i m p}=0.621 \pm 0.11 \mathrm{BW}$ and $\gamma=59.862 \pm 24.69 \mathrm{BW} / \mathrm{s}$. Fig. 6 shows the box plots of these results. The statistical analysis test performed provided a p-value $p<10^{-8}$, indicating that the null hypothesis can be rejected and it can be claimed that the RFS and FFS patterns result in significantly different values for $\gamma$ and $F_{i m p}$.

Table 1: Force indices and configuration-dependent performance indicators for RFS and FFS patterns in experiment series 1 . All values are expressed as mean \pm SD.

\begin{tabular}{llcc}
\hline & & RFS $(S I=15 \%)$ & FFS $(S I=73 \%)$ \\
\hline$F_{i m p}$ & {$[\mathrm{BW}]$} & $1.725 \pm 0.361$ & $0.621 \pm 0.11$ \\
$\gamma$ & {$[\mathrm{BW} / \mathrm{s}]$} & $444.8 \pm 36.42$ & $59.862 \pm 24.69$ \\
\hline$v_{t}^{-}(Q)$ & {$[\mathrm{m} / \mathrm{s}]$} & $0.61 \pm 0.21$ & $0.107 \pm 0.2116$ \\
$v_{n}^{-}(Q)$ & {$[\mathrm{m} / \mathrm{s}]$} & $-0.643 \pm 0.095$ & $-0.722 \pm 0.0881$ \\
$T_{c}^{-}$ & {$[\mathrm{J}]$} & $2.147 \pm 0.729$ & $0.759 \pm 0.242$ \\
$\xi$ & {$[\%]$} & $0.942 \pm 0.374$ & $0.214 \pm 0.122$ \\
$m_{e f f}$ & {$[\mathrm{~kg}]$} & $14.31 \pm 2.32$ & $1.632 \pm 0.49$ \\
$\chi$ & {$[\%]$} & $19.88 \pm 3.22$ & $2.27 \pm 0.68$ \\
$\mu_{c}$ & {$[-]$} & $0.245 \pm 0.01$ & $0.764 \pm 0.029$ \\
\hline
\end{tabular}

Regarding the motion kinematics, the value of the strike index with the RFS pattern was 
$S I=15 \%$, with $\beta=78^{\circ}$ and $l_{1}=9.6 \mathrm{~cm}$. For FFS, $S I=73 \%, \beta=0^{\circ}$, and $l_{1}=l_{H F}=13.2 \mathrm{~cm}$. Table 1 shows the values of the impact velocity components and the configuration-dependent performance indicators, along with the force indices obtained from GRF measurements. The full variation range of the indicators defined in Section 3 is shown in B.

Significant differences existed in the measured values of the kinematic and kinetic quantities between RFS and FFS cases. The value of $T_{c}^{-}$was higher for rear-foot strike, $T_{c}^{-}=2.15 \pm 0.73$ $\mathrm{J}$, compared with fore-foot running, in which $T_{c}^{-}=0.76 \pm 0.24 \mathrm{~J}$, with a p-value of $10^{-12}$. These results indicate that the kinetic energy associated with the SCM at the foot-ground contact onset was much higher for the RFS pattern than for the FFS one, not only in absolute terms but also in relative ones: $\xi=0.21 \pm 0.12 \%$ for FFS and $\xi=0.94 \pm 0.37 \%$ for RFS. These results are shown in Fig. 14.

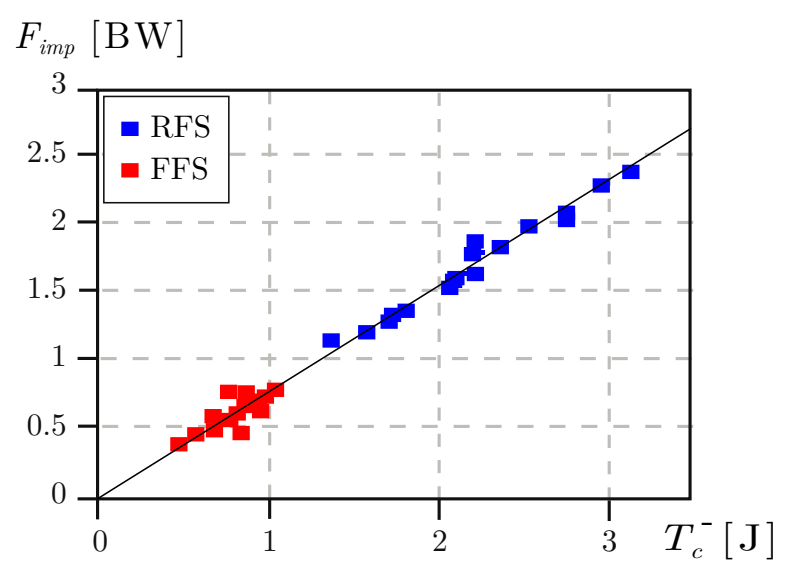

Figure 7: Correlation between $T_{c}^{-}$and $F_{i m p}$ for experiment series 1. Red dots represent FFS cases and blue dots RFS ones.

It was shown in [15] that $T_{c}^{-}$can be used as indicator of the peak impact force in rigid multibody impacts. Even though the foot-ground impact during running is not perfectly rigid, the system configuration does not change significantly between the contact onset and $t_{i m p}$; experimental data confirmed that $T_{c}^{-}$can be used as indicator of the maximum impact force in this case as well. Fig. 7 shows the correlation between $T_{c}^{-}$calculated from the kinematic data and $F_{i m p}$ evaluated from GRF measurements.

Regarding the effective mass terms, their values were in agreement with the impact intensity indicators based on kinetic energy. RFS resulted in $m_{\text {eff }}=14.31 \pm 2.32 \mathrm{~kg}$ and the corresponding dimensionless values normalized with the total body mass were $\chi=19.88 \pm 3.22 \%$. With FFS $m_{\text {eff }}=1.632 \pm 0.49 \mathrm{~kg}$ and $\chi=2.27 \pm 0.68 \%$ were obtained. Fig. 15 displays these 
results. Consistent results were observed for the critical friction coefficient $\mu_{c}$. A value of $\mu_{c}=$ $0.245 \pm 0.01$ was obtained for the RFS pattern, while $\mu_{c}=0.764 \pm 0.029$ for FFS cases, as shown in Fig. 16. For both $m_{e f f}$ and $\mu_{c}$ the statistical test confirmed the existence of significant differences between the two running patterns.

It must be noted that, although $m_{\text {eff }}$ can be used to show that RFS impacts are more intense than their FFS counterparts, a linear correlation with the peak impact force like the one between $T_{c}^{-}$and $F_{i m p}$ shown in Fig. 7 has not been found. Accordingly, it is not possible to use this indicator to directly quantify impact intensity.

\subsection{Experiment series 2: treadmill}

The performance indicators introduced in Section 3 were also evaluated using data from the second series of experiments. These data were obtained during the running motion of the subject on a treadmill, which ensured a constant running velocity. This, in turn, had a positive impact on data consistency and reduced the standard deviation of most measurements. For instance, the tangential and normal velocity components of the contact point were $v_{t}^{-}(Q)=0.774 \pm 0.075$ $\mathrm{m} / \mathrm{s}$ and $v_{n}^{-}(Q)=-0.581 \pm 0.040 \mathrm{~m} / \mathrm{s}$ in RFS experiments. For FFS running, the recorded values were $v_{t}^{-}(Q)=0.182 \pm 0.097 \mathrm{~m} / \mathrm{s}$ and $v_{n}^{-}(Q)=-0.716 \pm 0.047 \mathrm{~m} / \mathrm{s}$. Note that the these values are expressed in the belt reference frame. The value of the strike index observed during the experiments with RFS pattern was $S I=14 \%$, with $\beta=79^{\circ}$ and $l_{1}=9.6 \mathrm{~cm}$. For FFS, $S I=74 \%$, $\beta=0^{\circ}$, and $l_{1}=l_{H F}=13.2 \mathrm{~cm}$.

Table 2: Performance indicators for RFS and FFS patterns in experiment series 2. All values are expressed as mean \pm SD.

\begin{tabular}{llcc}
\hline & & RFS $(S I=14 \%)$ & FFS $(S I=74 \%)$ \\
\hline$v_{t}^{-}(Q)$ & {$[\mathrm{m} / \mathrm{s}]$} & $0.774 \pm 0.075$ & $0.182 \pm 0.097$ \\
$v_{n}^{-}(Q)$ & {$[\mathrm{m} / \mathrm{s}]$} & $-0.581 \pm 0.040$ & $-0.716 \pm 0.047$ \\
$T_{c}^{-}$ & {$[\mathrm{J}]$} & $1.8 \pm 0.4$ & $0.6 \pm 0.2$ \\
$\xi$ & {$[\%]$} & $0.94 \pm 0.24$ & $0.27 \pm 0.07$ \\
$m_{e f f}$ & {$[\mathrm{~kg}]$} & $13.83 \pm 1.12$ & $1.52 \pm 0.12$ \\
$\chi$ & {$[\%]$} & $19.21 \pm 1.55$ & $2.11 \pm 0.17$ \\
$\mu_{c}$ & {$[-]$} & $0.109 \pm 0.026$ & $0.797 \pm 0.028$ \\
\hline
\end{tabular}

The values of the performance indicators for this series of experiments are summarized in 
Table 2. Their box plots are depicted in B. The statistical analysis of the data resulted in a pvalue $p<10^{-4}$ for $T_{c}^{-}$and $\xi$; similar values were obtained for the effective mass and the critical coefficient of friction.

\subsection{Parametric analysis}

The effect on the indicators of the location of the foot-ground contact point was studied by means of a parametric analysis, which confirmed that the values of $T_{c}^{-}, \mu_{c}$, and $m_{\text {eff }}$ are very sensitive to changes in the strike index. Data from experiment series 2 were used for this analysis. For each running pattern, RFS and FFS, the average impact velocities and impact configuration were kept constant while varying the SI from 0 to $100 \%$ by modifying the values of $l_{1}$ and $\beta$.

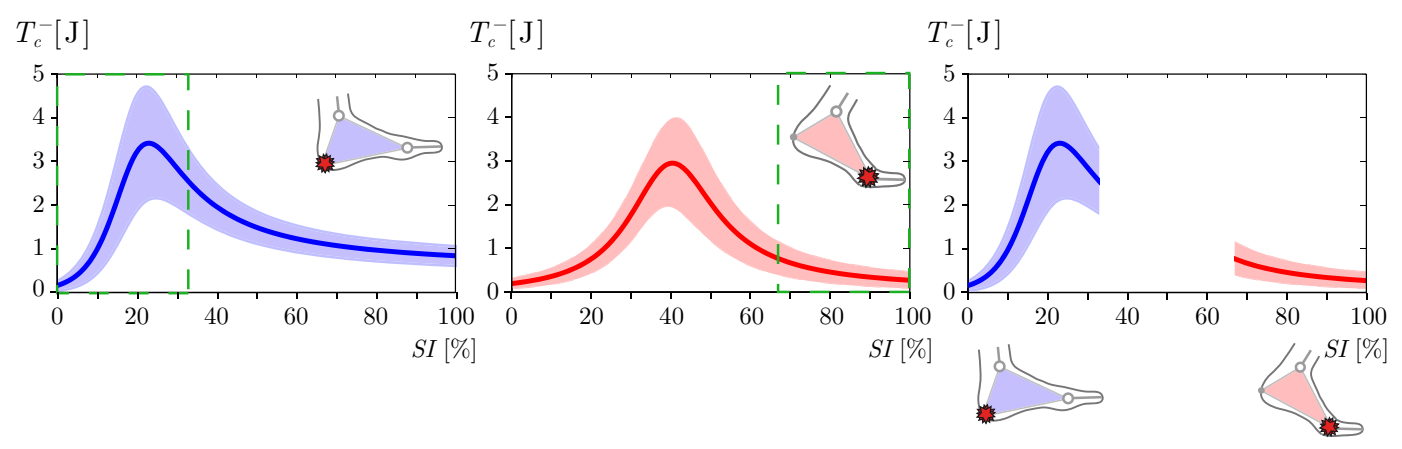

Figure 8: Evolution of $T_{c}^{-}$with the strike index.

Figure 8 shows the results for the effective pre-impact kinetic energy $T_{c}^{-}$. Three plots are included in the figure. The first one, drawn in blue, corresponds to the evaluation of the indicator assuming an RFS running pattern. The second plot, in red, shows the values obtained in the FFS case. Dashed boxes in these plots indicate the range of the SI in which each strike pattern is possible, $S I=0-33 \%$ for RFS and $S I=68-100 \%$ for FFS. The two valid regions are combined in the third plot, which shows the values that can be obtained in practice. The solid lines in Fig. 8 show the mean values of $T_{c}^{-}$for different values of the strike index, while the shaded areas around them denote two standard deviations across the mean values, which allow us to represent the 95\% confidence interval. Figs. 9 and 10 show the results of equivalent analyses conducted to evaluate the effect of SI on the effective mass and the critical coefficient of friction.

The use of the element $m_{e f f}$ of the effective mass matrix as indicator requires the assumption that all the DOFs of the system are locked and the related generalized velocities are zero, except 


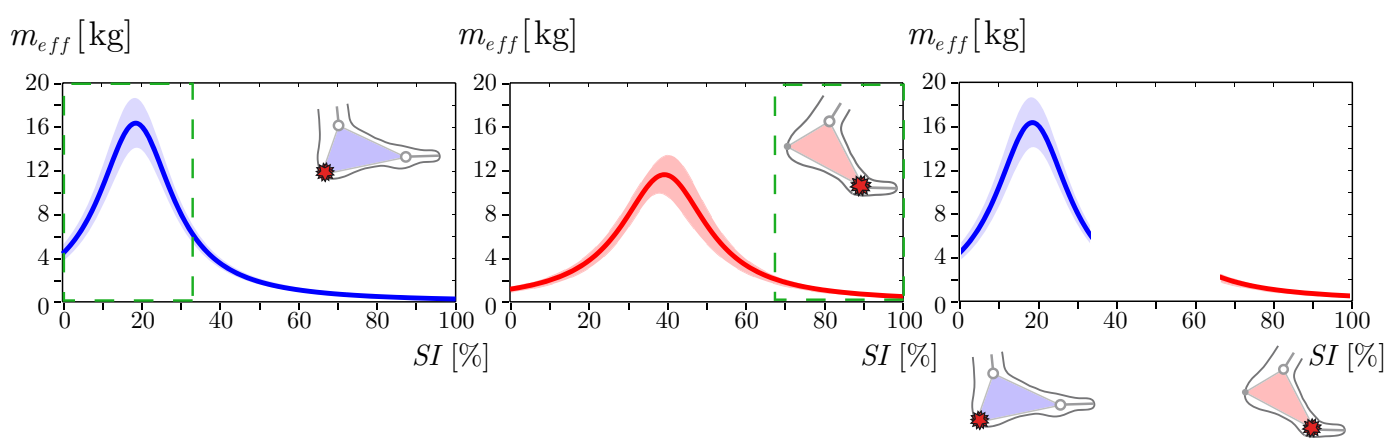

Figure 9: Evolution of $m_{e f f}$ with the strike index.

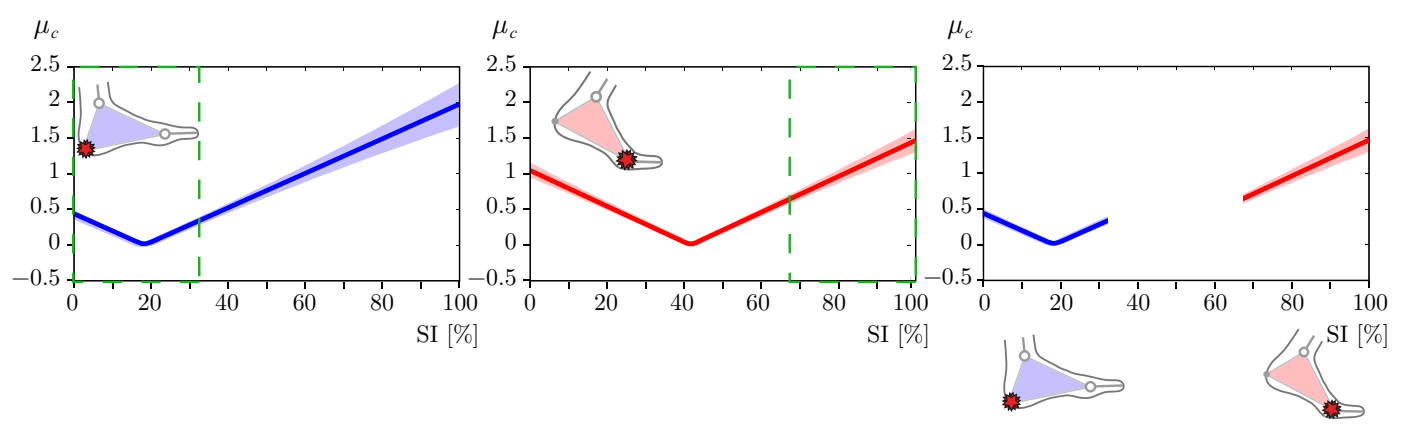

Figure 10: Evolution of $\mu_{c}$ with the strike index.

for the vertical component of the ankle velocity. The effect of this assumption on $T_{c}^{-}$is illustrated in Fig. 11, obtained making zero all the system velocities except for the $y$ component of point $P_{2}$, which kept the value recorded in the experiments. The effective pre-impact kinetic energy thus obtained is denoted by $\widetilde{T}_{c}^{-}$; the comparison of Figs. 8 and 11 shows that its value is significantly lower than $T_{c}^{-}$for the RFS case due to the horizontal component of the pre-impact ankle velocity.

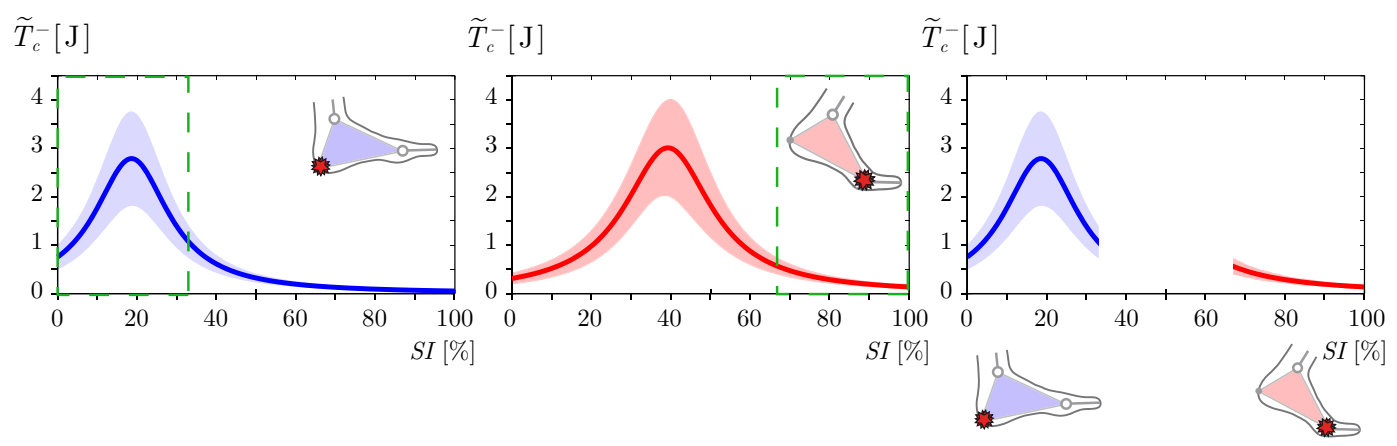

Figure 11: Evolution of $\widetilde{T}_{c}^{-}$with respect to the strike index. 


\subsection{Use of simplified models}

The above parametric studies were also performed using the simplified body models described in Section 2.3. The effective pre-impact kinetic energy and the effective mass matrix indicators obtained are shown in Figs. 12 and 13, respectively. The results obtained for the full body model, denoted by solid lines, were compared with the three partial models (Fig. 3), represented by dashed lines.
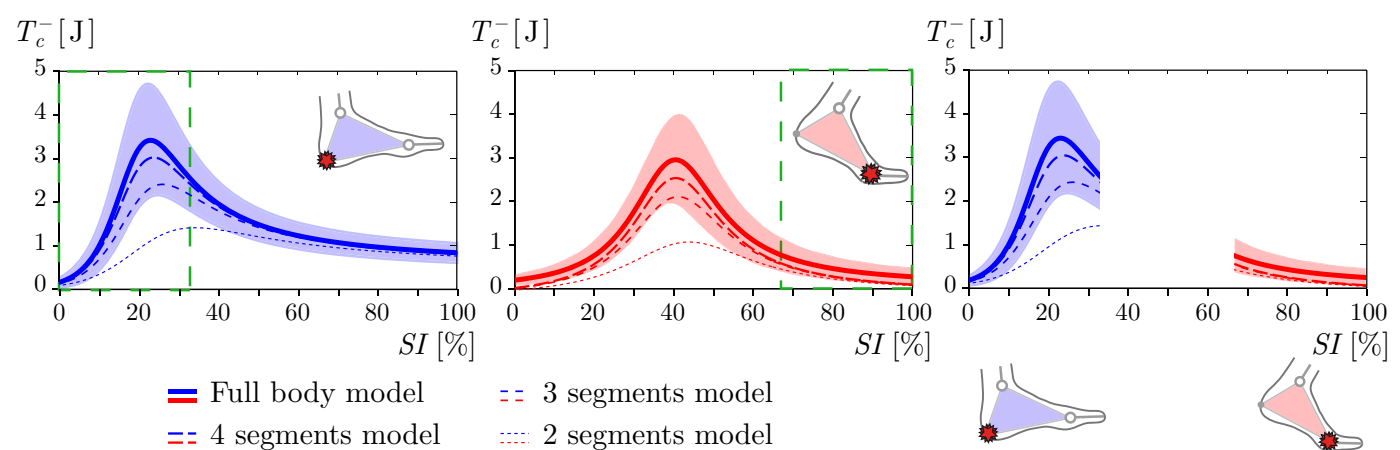

Figure 12: Evolution of $T_{c}^{-}$with respect to the strike index with different human body models.
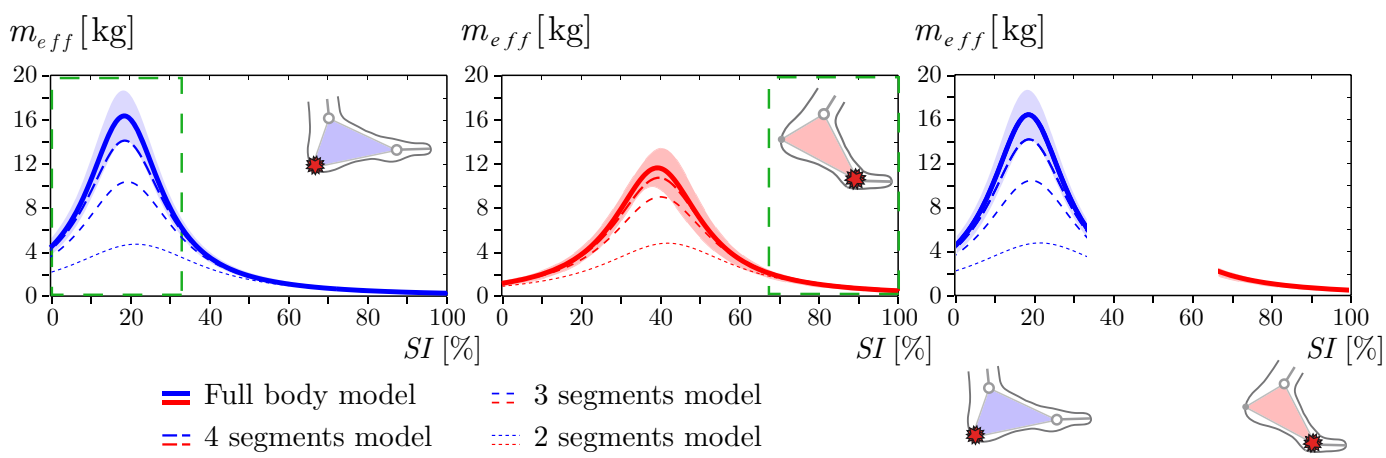

$=$ Full body model
$==4$ segments model

= $=3$ segments model

…. 2 segments model

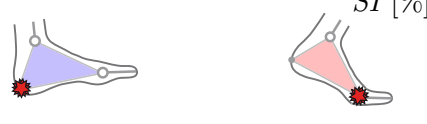

Figure 13: Evolution of $m_{e f f}$ with respect to the strike index with different human body models.

Regarding the critical coefficient of friction $\mu_{c}$, the use of partial human body models did not result in significant differences. Table 3 contains the mean and the standard deviation of $\mu_{c}$ evaluated with the $S I$ of the experimental cases, i.e., $S I=14 \%$ for RFS and $S I=74 \%$ for FFS, obtained with the four different human body models used. 
Table 3: Critical coefficient of friction $\mu_{c}$ obtained with the different human body models and the $S I$ measured during the experiments.

\begin{tabular}{lcc}
\hline & RFS $(S I=14 \%)$ & FFS $(S I=74 \%)$ \\
Model used & $\overline{\mu_{c}} \pm \mathrm{SD}$ & $\overline{\mu_{c}} \pm \mathrm{SD}$ \\
\hline Full body & $0.1090 \pm 0.026$ & $0.7970 \pm 0.028$ \\
4 segments & $0.1107 \pm 0.026$ & $0.8048 \pm 0.034$ \\
3 segments & $0.1149 \pm 0.025$ & $0.8022 \pm 0.038$ \\
2 segments & $0.1364 \pm 0.023$ & $0.7856 \pm 0.037$ \\
\hline
\end{tabular}

\section{Discussion}

The primary purpose of this study is to discuss the use of configuration-dependent performance indicators to characterize the dynamics in running impacts, specifically to evaluate differences between fore-foot and rear-foot impacts.

It has to be noted that the performance indicators described in this paper cannot be used to obtain force values. Instead, their main purpose is to predict the way in which changes in system configuration, velocities, and inertias distribution will affect them, i.e., whether they will result in an increase or decrease of the peak force during impact.

Two series of experiments, involving running on hard ground and on a treadmill respectively, were performed to assess these indicators. The presented indicators did not present significant differences between these two scenarios. Statistical tests were conducted to compare the indicators obtained on hard ground and on the treadmill; they yielded p-values above 0.1. Therefore, the null hypothesis, namely that data come from independent random samples from normal distributions with equal means, cannot be rejected at the default $5 \%$ level. This is in agreement with vertical GRF being very similar on hard ground and on treadmills, as reported in the literature [19], [36].

The effective pre-impact kinetic energy, $T_{c}^{-}$, was evaluated in experiments with both FFS and RFS running. Significant differences were detected between the two impact patterns. Results from the first series of experiments showed that $T_{c}^{-}$can be used to quantify the maximum impact force, $F_{i m p}$, developed at landing, as shown in Fig. 7. The peak force $F_{i m p}$ and loading rate $\gamma$ measured in this study remained in the range reported in [24]. Data obtained in the second experiment series confirmed that $T_{c}^{-}$with RFS was, in average, about three times higher than that with FFS. 
It was found that $T_{c}^{-}$is highly sensitive to the location of the foot-ground contact point. Fig. 8 illustrates this with the results of a parametric study, in which the strike index was varied from $S I=0$ to $S I=100 \%$, i.e., extreme rear-foot to extreme fore-foot treading, with both strike patterns. FFS runners typically contact the ground with the metatarsal area instead of the tip of the toes, which makes $S I \approx 75 \%$ in this case. With rear-foot strike, the contact point is just below the ankle, under the centre of mass of the foot and leg assembly, which results in $S I \approx 15 \%[4]$.

Fig. 8 shows that in extreme rear-foot cases, $0<S I<10 \%$, the effective pre-impact kinetic energy is similar to the fore-foot case values. Note that this effect was not observed neither in [24] nor in [22], since the authors of these papers used a strike index definition that differs from the one presented in Section 2.4. In these studies, SI ranged from 0 at the ankle point to $100 \%$ at the toes.

Figure 12 shows how the human body model affects the value of $T_{c}^{-}$. As expected, the simplified models described in Section 2.3 resulted in lower numerical values of the indicator, especially for the RFS case. Nevertheless, the four- and three-segment models kept the overall shape of the $T_{c}^{-}$vs. SI plot, which suggests that they can be used to predict trends of change of impact intensity with respect to variations of the strike index. Even with these simplified models, it is possible to capture the significant differences that exist between the values of $T_{c}^{-}$in the FFS and RFS patterns. Results obtained with the two-segment model, on the other hand, did not convey this information with the same clarity. This is in agreement with the results presented in [46], where it was concluded that, when indicators of foot impact intensity are used, it is not possible to achieve accurate results without modelling the motion of the thigh and the weight of the trunk.

The use of indicators based on effective mass terms is possible only under special circumstances. As discussed in Section 3.2, the velocity of the impact point $Q$ usually has a nonnegligible horizontal component at contact onset. As a consequence, the strike intensity cannot be fully characterized with a single component of the effective mass matrix, $m_{\text {eff }}$; all the components of the pre-impact velocity and the tensorial quantity $\mathbf{P}_{c}^{\mathrm{T}} \mathbf{M} \mathbf{P}_{c}$ should be considered to this end, as all of them contribute to the effective kinetic energy. However, most of the studies that have used the concept of effective mass modelled the foot as a single point and considered only the vertical component of its velocity at the instant immediately before the impact, e.g., [24], [7]. 
The results presented in [22] were obtained with a foot-shank model and the assumption that all pre-impact velocity components were zero, except for the vertical direction of the metatarsal joint. These can be compared to those obtained in this research with the two-segment model, shown in Fig. 13. It must be noted, however, that a different definition of the strike index, in which $S I=0$ corresponds to the ankle articulation, was used in [22].

Results in Fig. 13 confirm that, even with the above mentioned assumptions, $m_{\text {eff }}$ can be a meaningful indicator of foot-ground impact intensity. Simplified models, consisting of a limited number of segments, provide qualitative information about the evolution and trends of change of impact intensity with the strike index. In all cases, $m_{\text {eff }}$ values were higher for RFS than for FFS patterns. As shown in Fig. 11, the use of $\widetilde{T}_{c}^{-}$as indicator provides the same information as $m_{e f f}$, as only the vertical component of the ankle velocity is used to evaluate its value.

Experimental results confirmed that the critical friction coefficient $\mu_{c}$ is much lower for RFS than for FFS, as shown in Fig. 16. Rear-foot runners can avoid slip at the foot-ground interface with friction coefficients as low as $\mu_{c} \approx 0.3$. Fore-foot runners are more likely to slip under the same adherence conditions. Results in Fig. 16 also agree with the fact that, regardless of the strike pattern, no slip was observed during the experimental tests performed in this research. It must be mentioned that $\mu_{c}$ varies considerably depending on the strike index, as highlighted by Fig. 10. The use of partial body models did not result in significant changes in the indicator. As can be seen in Table 3, the average values for the partial models are inside the confidence interval of the full body model given by $\overline{\mu_{c}} \pm S D$.

Finally, it must be mentioned that the experimental results in this research were obtained with only one subject, a habitual RFS runner. The results for habitual FFS runners could differ from the ones captured in this study. Additionally, differences in running pattern across the runners population were not considered here. On the other hand, an advantage of using only one subject is that dynamic differences due to changes in the location of the markers or to estimated anthropometric parameters were avoided, since these parameters did not change among captures. Also, representing the human body with a complete 3D model would provide a more accurate simulation of the motion. Combining this model with an instrumented treadmill, where the contact force can be directly measured, would enable an additional validation of the indicators proposed in this research. Finally, increasing the level of detail in the foot model, e.g., using an articulated three-segment representation, could provide more insight about the impact dynamics, especially in the FFS case. 


\section{Conclusions}

This work presents new findings for the study of foot-ground impact during running. Three parametric indicators based on kinematic quantities were used to characterize impact intensity, namely the effective pre-impact kinetic energy $T_{c}^{-}$, the effective mass $m_{e f f}$, and the critical coefficient of friction $\mu_{c}$. The use of these indicators does not require any contact force measurements; they can be evaluated using experimental kinematic data obtained through motion capture. The presented indicators also represent a low computational cost alternative with respect to solving the forward- and inverse-dynamics problems. They enable the definition of a framework to characterize the relationship between the state of the system and impact intensity. Inference tests showed that $T_{c}^{-}, m_{e f f}$, and $\mu_{c}$ are statistically different between the RFS and the FFS cases. Moreover, experimental results showed that $T_{c}^{-}$can be used to quantify peak normal forces during foot-ground impact.

The analysis of the obtained indicator values indicated that the intensity of the collision when foot landing takes place following an RFS pattern is higher than in the case of FFS. This is in agreement with the results reported in the literature. Moreover, using indicators makes it possible to carry out parametric studies of the running gait to determine how variations in system variables affect foot landing impacts. The number of system parameters considered and the accuracy of the predictions can be increased when the indicators are evaluated with full human body models instead of simplified lower-limb ones. However, it was shown that the latter may be enough to capture the most relevant aspects of the running gait in some cases.

The conclusions of this study can be further validated in future research, using different treadmill velocity ranges and professional runners, with habitual RFS and FFS running patterns. These results would support the suitability of the indicators for clinical applications.

\section{Acknowledgements}

This research was supported in part by he Natural Sciences and Engineering Research Council of Canada. The second author was funded by the Spanish Ministry of Economy through its post-doctoral research program Juan de la Cierva, contract No. JCI-2012-12376. The support is gratefully acknowledged. 


\section{A Anthropometric parameters}

Table 4: Anthropometric data for the 2D model with twelve segments

\begin{tabular}{llccccc}
\hline & & & & & & \multicolumn{2}{c}{$\begin{array}{c}\text { Principal } \\
\text { moment of inertia }\end{array}$} \\
\cline { 4 - 5 } No. & Name & $L_{i}[\mathrm{~m}]$ & $x_{G i}^{\prime}[\mathrm{m}]$ & $y_{G i}^{\prime}[\mathrm{m}]$ & $m_{i}[\mathrm{~kg}]$ & $I_{G i}^{\prime}\left[10^{-2} \cdot \mathrm{kgm}^{2}\right]$ \\
\hline 1 & Trunk & 0.487 & 0.264 & 0.0 & 34.279 & 0.364 \\
2 & Head & 0.237 & 0.213 & 0.038 & 4.642 & 0.018 \\
3 & Right arm & 0.226 & 0.158 & 0.0 & 1.996 & 0.011 \\
4 & Right forearm & 0.227 & 0.105 & 0.0 & 1.407 & 0.005 \\
5 & Left arm & 0.198 & 0.138 & 0.0 & 1.743 & 0.010 \\
6 & Left forearm & 0.336 & 0.156 & 0.0 & 2.085 & 0.008 \\
7 & Right thigh & 0.397 & 0.138 & 0.0 & 7.864 & 0.082 \\
8 & Right shank & 0.438 & 0.163 & 0.0 & 3.588 & 0.038 \\
9 & Right hindfoot & 0.137 & 0.03 & -0.006 & 1.124 & 0.004 \\
10 & Left thigh & 0.400 & 0.139 & 0.0 & 7.933 & 0.083 \\
11 & Left shank & 0.450 & 0.167 & 0.0 & 3.692 & 0.039 \\
12 & Left hindfoot & 0.132 & 0.029 & -0.006 & 1.086 & 0.004 \\
\hline
\end{tabular}

Table 4 contains the anthropometric parameters used in this study. The position of the centre of mass of each segment $\left(x_{G i}^{\prime}, y_{G i}^{\prime}\right)$ is expressed using the local coordinate system with the origin at the proximal joint (See Fig. 2). The moments of inertia of the segments are calculated with respect to the local basis attached to its COM. It is assumed that the $\left(X^{\prime}, Y^{\prime}\right)$ axes are the principal directions of inertia and $\left(I_{G i}^{\prime}\right)$ are the principal moments of inertia about the COM.

\section{B Box plots results}

Figures 14-16 show the full variation range of the performance indicators defined in Section 3. Their likely range of variation and a typical value, the median, are represented with box plots. The shaded boxes correspond to results from experiment series 1, in which the subject was running on hard ground. The white boxes represent data obtained in the experiments conducted on the treadmill during series 2 . These figures highlight the reduced variability of the measurements when the subject runs on a treadmill, while confirming that in both series of experiments the values of the configuration-dependent indicators remained in similar ranges. 

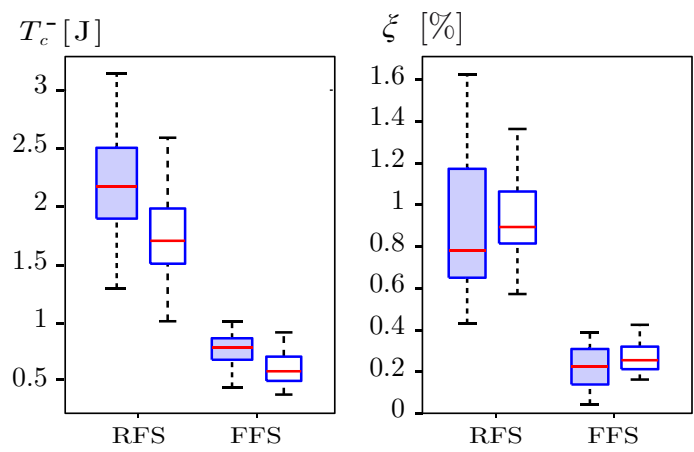

Figure 14: $T_{c}^{-}$and $\xi$ box plots for both foot strike patterns, RFS and FFS.
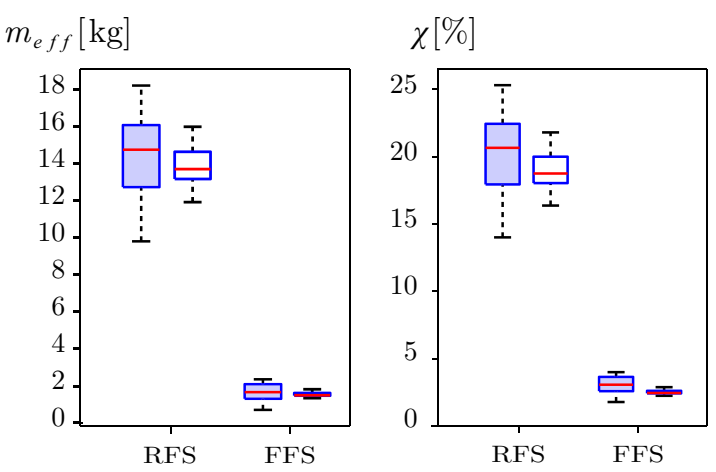

Figure 15: $m_{e f f}$ and $\chi$ box plots for both foot strike patterns, RFS and FFS.

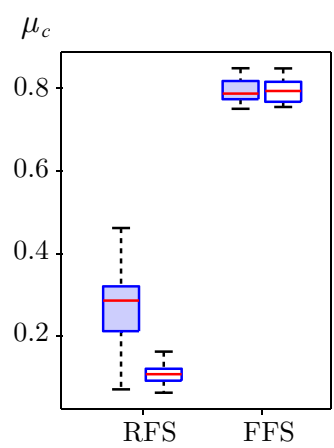

Figure 16: $\mu_{c}$ box plots for both foot strike patterns, RFS and FFS.

\section{References}

[1] Addison, B.J., Lieberman, D.E.: Tradeoffs between impact loading rate, vertical impulse and effective mass for walkers and heel strike runners wearing footwear of varying stiffness. Journal of Biomechanics 48(7), 1318 - 1324 (2015). DOI doi:10.1016/j.jbiomech. 2015.01.029

[2] Alonso, F.J., Castillo, J.M.D., Pintado, P.: Application of singular spectrum analysis to the 
smoothing of raw kinematic signals. Journal of Biomechanics 38(5), 1085-1092 (2005). DOI 10.1016/j.jbiomech.2004.05.031

[3] Alonso, F.J., Cuadrado, J., Lugrís, U., Pintado, P.: A compact smoothing-differentiation and projection approach for the kinematic data consistency of biomechanical systems. Multibody System Dynamics 24(1), 67-80 (2010). DOI 10.1007/s11044-010-9191-1

[4] Altman, A.R., Davis, I.S.: A kinematic method for footstrike pattern detection in barefoot and shod runners. Gait \& Posture 35(2), 298 - 300 (2012). DOI http://dx.doi.org/10. 1016/j.gaitpost.2011.09.104

[5] Belli, A., Bui, P., Berger, A., Geyssant, A., Lacour, J.R.: A treadmill ergometer for threedimensional ground reaction forces measurement during walking. Journal of Biomechanics 34(1), 105-112 (2001). DOI 10.1016/S0021-9290(00)00125-1

[6] Cavanagh, P.R., Lafortune, M.A.: Ground reaction forces in distance running. Journal of Biomechanics 13(5), 397-406 (1980). DOI 10.1016/0021-9290(80)90033-0

[7] Chi, K.J., Schmitt, D.: Mechanical energy and effective foot mass during impact loading of walking and running. Journal of Biomechanics 38(7), 1387-1395 (2005). DOI 10.1016/ j.jbiomech.2004.06.020

[8] Clark, K.P., Ryan, L.J., Weyand, P.G.: Foot speed, foot-strike and footwear: linking gait mechanics and running ground reaction forces. Journal of Experimental Biology 217, 2037-2040 (2014). DOI 10.1242/jeb.099523

[9] De Wit, B., De Clerq, D., Aerts, P.: Biomechanical analysis of the stance phase during barefoot and shod running. Journal of Biomechanics 33(3), 269-278 (2000). DOI 10. 1016/S0021-9290(99)00192-X

[10] Divert, C., Mornieux, G., Baur, H., Mayer, F., Belli, A.: Mechanical comparison of barefoot and shod running. International Journal of Sports Medicine 26(7), 593-598 (2005). DOI $10.1055 / \mathrm{s}-2004-821327$

[11] Farley, C.T., González, O.: Leg stiffness and stride frequency in human running. Journal of Biomechanics 29(2), 181 - 186 (1996). DOI http://dx.doi.org/10.1016/0021-9290(95) 00029-1 
[12] Font-Llagunes, J.M., Barjau, A., Pàmies-Vilà, R., Kövecses, J.: Dynamic analysis of impact in swing-through crutch gait using impulsive and continuous contact models. Multibody System Dynamics 28(3), 257-282 (2012). DOI 10.1007/s11044-011-9300-9

[13] Font-Llagunes, J.M., Kövecses, J.: Dynamics and energetics of a class of bipedal walking systems. Mechanism and Machine Theory 44(11), 1999-2019 (2009). DOI 10.1016/j. mechmachtheory.2009.05.003

[14] Gerritsen, K.G., van den Bogert, A.J., Nigg, B.M.: Direct dynamics simulation of the impact phase in heel-toe running. Journal of Biomechanics 28(6), 661-668 (1995). DOI 10. 1016/0021-9290(94)00127-P

[15] González, F., Kövecses, J., Font-Llagunes, J.M.: Load assessment and analysis of impacts in multibody systems. Multibody System Dynamics 38(1), 1-19 (2016). DOI 10.1007/ s11044-015-9485-4

[16] Hamner, S.R., Seth, A., Delp, S.L.: Muscle contributions to propulsion and support during running. Journal of Biomechanics 43(14), 2709 - 2716 (2010). DOI http://dx.doi.org/ 10.1016/j.jbiomech.2010.06.025

[17] Hanson, N.J., Berg, K., Deka, P., Meendering, J.R., Ryan, C.: Oxygen cost of running barefoot vs. running shod. International Journal of Sports Medicine 32(6), 401-406 (2011). DOI 10.1055/s-0030-1265203

[18] Hirschkorn, M., Kövecses, J.: The role of the mass matrix in the analysis of mechanical systems. Multibody System Dynamics 30(4), 397-412 (2013). DOI 10.1007/ s11044-013-9369-4

[19] Kluitenberg, B., Bredeweg, S.W., Zijlstra, S., Zijlstra, W., Buist, I.: Comparison of vertical ground reaction forces during overground and treadmill running. a validation study. BMC Musculoskeletal Disorders 13(1), 235 (2012). DOI 10.1186/1471-2474-13-235

[20] Kövecses, J.: Dynamics of mechanical systems and the generalized free-body diagram part I: General formulation. Journal of Applied Mechanics 75(6, paper 061012), 1 - 12 (2008). DOI 10.1115/1.2965372

[21] Kövecses, J., Font-Llagunes, J.M.: An eigenvalue problem for the analysis of variable topology mechanical systems. Journal of Computational and Nonlinear Dynamics 4(3, paper 031006), 1-9 (2009). DOI 10.1115/1.3124784 
[22] Kövecses., J., Kovács, L.: Foot impact in different modes of running: mechanisms and energy transfer. Procedia IUTAM 2, 101-108 (2011). DOI 10.1016/j.piutam.2011.04.011

[23] Laughton, C.A., Davis, I.M., Hamill, J.: Effect of strike pattern and orthotic intervention on tibial shock during running. Journal of Applied Biomechanics 19(2), 153-168 (2003). DOI 10.1123/jab.19.2.153

[24] Lieberman, D.E., Venkadesan, M., Werbel, W.A., Daoud, A.I., D’Andrea, S., Davis, I.S., Mang'Eni, R.O., Pitsiladis, Y.: Foot strike patterns and collision forces in habitually barefoot versus shod runners. Nature 463, 531-535 (2010). DOI 10.1038/nature08723

[25] Lopes, D., Neptune, R., Ambrósio, J., Silva, M.: A superellipsoid-plane model for simulating foot-ground contact during human gait. Computer Methods in Biomechanics and Biomedical Engineering 19(9), 954-963 (2016). DOI 10.1080/10255842.2015.1081181

[26] Low, D.C., Dixon, S.J.: Footscan pressure insoles: Accuracy and reliability of force and pressure measurements in running. Gait \& Posture 32(4), 664-666 (2010). DOI 10.1016/ j.gaitpost.2010.08.002

[27] Mahboobin, A., Cham, R., Piazza, S.J.: The impact of a systematic reduction in shoe-floor friction on heel contact walking kinematics-a gait simulation approach. Journal of Biomechanics 43(8), 1532-1539 (2010). DOI 10.1016/j.jbiomech.2010.01.040

[28] Maiwald, C., Grau, S., Krauss, I., Mauch, M., Axmann, D., Horstmann, T.: Reproducibility of plantar pressure distribution data in barefoot running. Journal of Applied Biomechanics 24(1), 14-23 (2008). DOI 10.1123/jab.24.1.14

[29] McDougall, C.: Born to run: a hidden tribe, superathletes, and the greatest race the world has never seen, 1st edn. Knopf, New York (2009)

[30] McMahon, T.A., Cheng, G.C.: The mechanics of running: How does stiffness couple with speed? Journal of Biomechanics 23, Supplement 1, 65 - 78 (1990). DOI http://dx.doi. org/10.1016/0021-9290(90)90042-2

[31] Neptune, R.R., Wright, I.C., van den Bogert, A.J.: A method for numerical simulation of single limb ground contact events: Application to heel-toe running. Computer Methods in Biomechanics and Biomedical Engineering 3(4), 321-334 (2000). DOI $10.1080 / 10255840008915275$ 
[32] Nigg, B.M. (ed.): Biomechanics of running shoes. Human Kinetics, Champaign, IL, USA (1986)

[33] O'Connor, C.M., Thorpe, S.K., O’Malley, M.J., Vaughan, C.L.: Automatic detection of gait events using kinematic data. Gait \& Posture 25(3), 469-474 (2007). DOI 10.1016/j. gaitpost.2006.05.016

[34] Pàmies-Vilà, R.: Application of multibody dynamics techniques to the analysis of human gait. Ph.D. thesis, Universitat Politècnica de Catalunya (2012). URL http://www . tdx . cat/handle/10803/123774

[35] Pàmies-Vilà, R., Font-Llagunes, J., Lugrís, U., Cuadrado, J.: Parameter identification method for a three-dimensional foot-ground contact model. Mechanism and Machine Theory 75, 107-116 (2014). DOI 10.1016/j.mechmachtheory.2014.01.010

[36] Riley, P.O., Dicharry, J., Franz, J.R., Casey, K.D.: A kinematics and kinetic comparison of overground and treadmill running. Medicine \& Science in Sports \& Exercise 40(6), 10931100 (2008). DOI 10.1249/MSS.0b013e3181677530

[37] Robbins, S.E., Gouw, G.J., Hanna, A.M.: Running-related injury prevention through innate impact-moderating behavior. Medicine \& Science in Sports \& Exercise 21(2), 130-139 (1989)

[38] Robbins, S.E., Hanna, A.M.: Running-related injury prevention through barefoot adaptations. Medicine \& Science in Sports \& Exercise 19(2), 148-156 (1987)

[39] Rodrigo, S.E., Ambrósio, J.A.C., Tavares da Silva, M.P., Penisi, O.H.: Analysis of human gait based on multibody formulations and optimization tools. Mechanics Based Design of Structures and Machines 36(4), 446-477 (2008). DOI 10.1080/15397730802425497

[40] Seyfarth, A., Geyer, H., Günther, M., Blickhan, R.: A movement criterion for running. Journal of Biomechanics 35(5), 649 - 655 (2002). DOI http://dx.doi.org/10.1016/ S0021-9290(01)00245-7

[41] Silva, M.P.T., Ambrósio, J.: Kinematic data consistency in the inverse dynamic analysis of biomechanical systems. Multibody System Dynamics 8(2), 219-239 (2002). DOI 10. 1023/A:1019545530737 
[42] Squadrone, R., Gallozzi, C.: Biomechanical and physiological comparison of barefoot and two shod conditions in experienced barefoot runners. The Journal of Sports Medicine and Physical Fitness 49(1), 6-13 (2009)

[43] Vaughan, C.L., Davis, B.L., O'Connor, J.C.: Dynamics of Human Gait, 2nd edn. Kiboho, Cape Town, South Africa (1999)

[44] Wright, I., Neptune, R., van den Bogert, A., Nigg, B.: Passive regulation of impact forces in heel-toe running. Clinical Biomechanics 13(7), 521 - 531 (1998). DOI http://dx.doi.org/ 10.1016/S0268-0033(98)00025-4

[45] Yong, J.R., Silder, A., Delp, S.L.: Differences in muscle activity between natural forefoot and rearfoot strikers during running. Journal of biomechanics 47(15), 3593-3597 (2014). DOI 10.1016/j.jbiomech.2014.10.015

[46] Zelei, A., Bencsik, L., Kovács, L., Stépán, G.: Energy efficient walking and running - impact dynamics based on varying geometric constraints. In: 12th Conference on Dynamical Systems Theory and Applications, pp. 259-270. Lódź, Poland (2013) 